\title{
Acute cholecystitis - a cohort study in a real-world clinical setting (REWO study, NCT02796443)
}

This article was published in the following Dove Press journal: Pragmatic and Observational Research

Jennifer Blythe

Eva Herrmann ${ }^{2}$

Dominik Faust ${ }^{3}$

Stephan Falk ${ }^{4}$

Tina Edwards-Lehr ${ }^{5}$

Florian Stockhausen ${ }^{6}$

Ernst Hanisch ${ }^{5}$

Alexander Buia ${ }^{5}$

'Department of Internal Medicine, Bürgerhospital Frankfurt, Frankfurt, Germany; ${ }^{2}$ Institute for Biostatistics and Mathematical Modelling,

University Hospital Goethe University,

Frankfurt, Germany; ${ }^{3}$ Department of Gastroenterology, Hepatology and Infectious Disease, Asklepios Klinik Langen, Langen, Germany; ${ }^{4}$ OptiPath Pathology Associates Frankfurt, Frankfurt, Germany; ${ }^{5}$ Department of Visceral and Thoracic Surgery, Asklepios Klinik Langen, Langen, Germany; ${ }^{6}$ Department of Surgery, Hospital zum Heiligen Geist, Frankfurt, Germany
Correspondence: Alexander Buia Asklepios Klinik Langen, Röntgenstr 20, 63220 Langen, Germany

$\mathrm{Tel}+4906 \quad 03912 \quad 1309$

Fax +4906 1039121814

Email a.buia@t-online.de
Background: For decades, the optimal timing of surgery for acute cholecystitis has been controversial. Recent meta-analyses and population-based studies favor early surgery. One recent large randomized trial has demonstrated that a delayed approach increases morbidity and cost compared to early surgery within 24 hours of hospital admission. Since cases of severe cholecystitis were excluded from this trial, we argue that these results do not reflect real-world clinical situations. From our point of view, these results were in contrast to the clinical experience with our patients; so, we decided to analyze critically all our patients with the null hypothesis that the patients treated with a delayed cholecystectomy after an acute cholecystitis have a similar or even better outcome than those treated with an early operative approach.

Patients and methods: We retrospectively analyzed clinical data from all patients with cholecystectomies in the period between January 2006 and September 2015. A total of 1,723 patients were categorized into four groups: early $(n=138)$ : urgent surgery of patients with acute cholecystitis within the first 72 hours of the onset of symptoms; intermediate ( $n=297)$ : surgery of patients with acute cholecystitis within an average of 10 days after the onset of symptoms; delayed $(n=427)$ : initial non-surgical treatment of acute cholecystitis with surgery performed within 6-12 weeks of the onset of symptoms; and elective $(n=868)$ : cholecystectomy within a symptom-free interval of choice in patients with symptomatic cholecystolithiasis without signs of acute cholecystitis.

Results: In a real-world scenario, early/intermediate cholecystectomy in acute cholecystitis was associated with a significant increase in morbidity and mortality (Clavien-Dindo score) compared to a delayed approach with surgery performed 6-12 weeks after the onset of symptoms. The adjusted linear rank statistics showed a decrease in the complication score with values of 2.29 in the early group, 0.48 in the intermediate group, -0.26 in the delayed group and -2.12 in the elective group. The results translate into a continuous decrease of the complication score from early over intermediate and delayed to the elective group.

Conclusion: These results demonstrate that delayed cholecystectomy can be performed safely. In cases with severe cholecystitis, early and/or intermediate approaches still have a relatively high risk of morbidity and mortality.

Keywords: acute cholecystitis, early cholecystectomy, delayed cholecystectomy, gallstone disease

\section{Introduction}

Although the true incidence of acute cholecystitis is unknown, ${ }^{1}$ some epidemiological data are available: in the UK, during the period from April 2003 to March 2004, 25,743 patients were admitted as an emergency with acute gallbladder disease. ${ }^{2}$ In Germany, 
64,000 patients with acute cholecystitis are hospitalized each year. ${ }^{3}$ For decades, the surgical community has debated the pros and cons of early vs delayed cholecystectomy for this disease. The discussion started early in the last century when Kehr reported his personal experience with gallbladder surgery. ${ }^{4}$ The plea for an early operation within the first day or two after the onset of biliary colic can be dated back to Graham et $\mathrm{al}^{5}$ in 1938 . They reported a mortality rate of $3.59 \%$, whereas others using a delayed approach reported a mortality rate of $10 \%$. In the era of evidence-based medicine, a Cochrane meta-analysis 2013 indicated that early laparoscopic cholecystectomy (ELC) seems safe and may shorten the total hospital stay. ${ }^{6}$ However, most trials carried a relatively high bias. Another meta-analysis of randomized studies showed that ELC results in decreased wound infections, a shorter total length of stay and decreased costs with no differences in mortality, bile duct injuries, bile leaks and conversions. ${ }^{7}$ In a meta-analysis of case-control studies, the same authors came to the conclusion that ELC is clearly superior to delayed laparoscopic cholecystectomy. ${ }^{8}$ In a recent meta-analysis, it was concluded that ELC appears to be as safe and effective as delayed laparoscopic cholecystectomy. ${ }^{9}$ Finally, the largest randomized trial so far has provided compelling evidence of the superiority of ELC, similar to the population-based study by de Mestral et al. ${ }^{10,11}$ Since our clinical experience has differed from these studies, we argue that the conclusions of the latter two studies, which excluded severe cases of cholecystitis, ${ }^{12}$ may not reflect real-world clinical scenarios. Therefore, we performed a retrospective analysis of all our patients who underwent cholecystectomy using the Clavien-Dindo Complication Score and the histologic examination results (the gold standard for the diagnosis $^{13}$ along with STROBE guidelines [STrengthening the Reporting of OBservational studies in Epidemiology]). ${ }^{14}$

\section{Patients and methods}

\section{Study design and overview}

This retrospective cohort study analyzed a total of 1,723 patients, that is, all patients affected with acute cholecystitis, or symptomatic cholecystolithiasis who underwent cholecystectomy in the Department of Visceral and Thoracic Surgery, Asklepios Hospital Langen, Germany from January 2006 through September 2015. The study has been approved by the ethics committee of the Hesse State Chamber of Physicians (No. FF8/2015). No extra patient consent was required because of the retrospective study design; data confidentiality and permission of data review were provided in the hospital admission consent. The study was registered on ClinicalTrials.gov (identifier: NCT02796443).

\section{Setting}

Asklepios Hospital Langen is a 400-bed academic teaching hospital of the Goethe-University Frankfurt/Main. Six operating rooms (ORs) are available for elective cases. There is no reserved "red" room for emergency cases. Regular operations are performed from 7.30 am to $3.30 \mathrm{pm}$; thereafter, two ORs are available until $6.00 \mathrm{pm}$ and one OR for the remaining time. Emergency cases during the day are organized by canceling elective operations. Over all disciplines, the hospital has a proportion of $70 \%$ emergency cases. A 12-bed interdisciplinary intensive care unit is available 24 hours.

Each year, 150-200 endoscopic retrograde cholangiopancreaticographies (ERCPs) are performed by the Department of Gastroenterology. The main focus of the Department of Visceral and Thoracic Surgery is minimally invasive techniques. The staff consists of one surgical chair, four senior surgeons and nine surgical residents. Cholecystectomy for acute cholecystitis is always performed by a senior physician or the chair. Indications for ERCP and for the treatment of acute cholecystitis are standardized by department policy. All cholecystectomies conform to a treatment protocol in accordance with the requirements of a peer review project of the Hesse State Chamber of Physicians, Germany (Supplementary material 1).

In addition, the department is a regional study center of CHIR-Net, a surgical study network, and is certified by CAMIC (surgical working group minimally invasive surgery) of the German Association of General and Visceral Surgery.

\section{Participants and variables}

Patients with acute cholecystitis are primarily referred to the Department of Gastroenterology. In severe cases, consultation by a senior surgeon is obtained within 24 hours. The timing of cholecystectomy is determined by the attending surgeon based on clinical findings. The diagnosis of acute cholecystitis is based on the presence of at least three of the following symptoms: upper right quadrant abdominal pain, positive Murphy's sign, leukocytosis and/or fever.

All patients with biliary colic without signs of acute cholecystitis were classified as having symptomatic cholecystolithiasis and were scheduled for elective cholecystectomy.

We categorized the patients into four groups as described in methods above.

All defined study variables were located on a standardized datasheet. Patient data were reviewed and extracted by means of a detailed chart analysis and entered into an electronic data bank using Microsoft Excel.

The following parameters were included: age, sex, comorbidities, physical status American Society of Anesthesiologist 
Risk Score (ASA-score), onset of symptoms, open or laparoscopic surgery, conversion rate, duration of surgery, mortality, complication rates (Clavien-Dindo Complication Score ${ }^{15}$ and length of pre- and postoperative hospital stay and pathologic diagnosis.

Data quality audit (Supplementary material 2A-C) was performed randomly every 3 months. A clinical pathway for symptomatic cholecystolithiasis was implemented and systematically audited.

\section{Study size and data sources}

The study comprised all patients of the Department of Visceral and Thoracic Surgery with acute cholecystitis and symptomatic cholecystolithiasis $(\mathrm{N}=1,723)$. The patients were identified by ICD/operations and procedure codes via the electronic medical information system. All paper charts were then retrieved from the archive and analyzed. Data collection took place from October 2014 until December 2015, and data analysis from January 2016 until January 2017.

\section{Operation}

Our standard operative technique for laparoscopic cholecystectomy is the four-port technique, and details of our technique are provided in Supplementary material 3. The technique of single-port laparoscopic cholecystectomy is also allowed, and its safety has been reported previously. ${ }^{16}$ In cases of choledocholithiasis, a preoperative ERCP is performed. Intraoperative cholangiography is not used. A primary open approach is only chosen in patients with multiple prior abdominal operations. The WHO Surgical Checklist is implemented and its use audited.

\section{Statistical analysis}

All data were statistically analyzed by using the nonparametric Kruskal-Wallis test, the Wilcoxon-Mann-Whitney test and the chi-squared test as appropriate. Primary analysis was the comparison of Clavien-Dindo Complication Score between the four groups stratified by histology, severity of histologic changes and ASA-scores with a stratified form of a Kruskal-Wallis test. Such an analysis allows confounding bias to be avoided with respect to the most important confounders similar to but even more effective than an adjustment in a multivariate regression analysis. Statistical analysis was performed with $\mathrm{R}$ software version 3.3.2 (R Foundation for Statistical Computing, Vienna, Austria) using the package coin version 1.1-2. ${ }^{17}$

All tests were two-sided, and a $P$-value $<0.05$ was considered significant.

\section{Results}

The quality of data documentation by physicians reached $100 \%$ in almost every audit (Supplementary material 2C). The audit of the implemented clinical pathway for elective cholecystectomy demonstrates an adherence to the pathway of over $90 \%$.

In total, 1,723 patients of the Department of Visceral and Thoracic Surgery underwent surgery for acute and symptomatic cholecystolithiasis. In this population, 60.99\% $(n=1,051)$ of the patients were female and $39.01 \%(n=672)$ were male.

According to the ASA-score, overall, 14.80\% $(n=255)$ of the patients were classified as ASA I, 77.19\% $(n=1,330)$ as ASA II, 7.37\% $(n=127)$ as ASA III and 0.64\% $(n=11)$ as ASA IV.

Overall, $83.87 \%$ of the patients $(n=1,445)$ were operated on using a four-port-laparoscopic approach and $11.02 \%$ $(n=190)$ using a single-port laparoscopy. Fifty-three patients were operated on primarily with an open access and 35 patients had to be converted from laparoscopic to an open procedure. The standardized operative technique only differed in the approach to the abdomen and the fundamental steps of the operation were the same in both laparoscopic groups.

The mean operating time for all groups was 63 minutes.

The overall conversion rate to open surgery was $2.03 \%$ $(n=35)$. Primary open surgical access was performed in 53 patients $(3.07 \%)$.

Table 1 shows the descriptive data of the groups. There were significant differences in ASA classification $(P<0.0001)$, duration of operation $(P<0.0001)$, hospitalization after cholecystectomy $(P<0.0001)$, four-port laparoscopy $(P<0.0001)$ and single-port access $(P<0.0001)$ between the study groups.

On comparing early vs intermediate vs delayed vs elective populations, age was found to be significantly different with the oldest population in the early group and the youngest in the elective group $(P<0.0001)$. Primary open access was significantly more frequent in the early compared to the intermediate group $(P=0.013)$.

The intermediate group ( $n=297)$ comprised 34 patients in whom a delayed approach was chosen. As the clinical signs did not improve, they were operated on after a mean of 6 days from the day of hospitalization. These patients had an onset of symptoms 2 days (mean) before hospitalization.

In total, 29 patients in the intermediate group could be discharged after a successful conservative treatment, but were readmitted because of recurrent symptoms.

Ninety-six patients in this group had surgery after a mean hospital stay of 7 days ( 2 days after the onset of symptoms) because these patients were in need of diagnostic procedures 
Table I Patients demographics, ASA classification, surgical procedures and hospitalization

\begin{tabular}{|c|c|c|c|c|}
\hline & $\begin{array}{l}\text { Early, } \\
n=138\end{array}$ & $\begin{array}{l}\text { Intermediate, } \\
\mathrm{n}=297\end{array}$ & $\begin{array}{l}\text { Delayed, } \\
n=427\end{array}$ & $\begin{array}{l}\text { Elective, } \\
\mathrm{n}=86 \mathrm{I}\end{array}$ \\
\hline Age, mean (SD), years & $58(17)$ & $57(17)$ & $56(16)$ & $54(16)$ \\
\hline Female sex, n (\%) & $69(50)$ & $174(58.8)$ & $243(56.9)$ & $565(65.6)$ \\
\hline Male sex, n (\%) & $69(50)$ & $123(4 \mid .2)$ & $184(43.1)$ & $296(34.4)$ \\
\hline ASA I, n (\%) ${ }^{\mathrm{a}}$ & II (7.9) & $24(8.1)$ & $57(13.3)$ & $163(18.9)$ \\
\hline ASA II, n (\%) ${ }^{\mathrm{a}}$ & 99 (7I.7) & $236(79.7)$ & $335(78.5)$ & $660(76.7)$ \\
\hline ASA III, n (\%) ${ }^{\mathrm{a}}$ & $19(13.8)$ & $35(11.5)$ & $35(8.2)$ & $38(4.4)$ \\
\hline ASA IV, n (\%) & $9(6.5)$ & $2(0.5)$ & $0(0)$ & $0(0)$ \\
\hline Four-port laparoscopy, ${ }^{\mathrm{a}} \mathrm{n}(\%)$ & $112(81.2)$ & $256(86.1)$ & $360(84.3)$ & $717(83.3)$ \\
\hline Single-port laparoscopy, ${ }^{\mathrm{a}} \mathrm{n}(\%)$ & $4(2.9)$ & II (3.7) & $48(\mathrm{II} .2)$ & $127(14.8)$ \\
\hline Conversion rate to open surgery, $\mathrm{n}(\%)$ & $7(5.1)$ & $13(4.4)$ & $6(1.4)$ & $9(1.0)$ \\
\hline Primarily open surgery, n (\%) & $15(10.9)$ & $17(5.7)$ & $13(3.1)$ & $8(0.9)$ \\
\hline Operation time, ${ }^{a}$ mean (SD), minutes & $68(40)$ & 6I (29) & $68(34)$ & $53(23)$ \\
\hline Duration of hospitalization after cholecystectomy, ${ }^{a}$ mean (SD), days & $6(6)$ & $5(5)$ & $4(2)$ & $3(2)$ \\
\hline Duration of hospitalization before cholecystectomy, mean (SD), days & $\mathrm{I}(\mathrm{I})$ & $4(5)$ & $0(0)$ & $0(0)$ \\
\hline
\end{tabular}

Notes: Early: Surgery performed within 72 hours of the onset of symptoms. Intermediate: Surgery performed within 10 days on average from the onset of symptoms (for detailed description, see the "Results" section: descriptive data). Delayed: Surgery performed in symptom-free interval (6-12 weeks after the onset of symptoms). Elective: Surgery performed electively in symptomatic cholecystolithiasis without signs of acute cholecystitis. ASA physical status classification system: ASA I: a normal healthy patient; ASA II: a patient with mild systemic disease; ASA III: a patient with severe systemic disease; ASA IV: a patient with severe systemic disease that is a constant threat to life. ${ }^{a} P<0.0001$.

Abbreviation: ASA, American Society of Anesthesiologists.

(ERCP, gastroscopy, cardiology procedures) and had comorbidities (acute pancreatitis, coagulopathy). These patients also had an onset of symptoms of 2 days (mean) before hospitalization.

In total, 138 patients with a mean history of 9 days after the onset of symptoms were operated upon after a clinical re-evaluation following a 3-day hospital stay.

The histologic diagnoses are shown in Table 2. The majority of patients who were diagnosed with severe cholecystitis with abscess, empyema, necrosis or covered perforation were in the early and intermediate groups.

The complications in the defined groups were classified according to the Clavien-Dindo Complication Score.

This complication score is divided into six tiers. Grade 0 denotes no complications; Grade 1 denotes any deviation from the normal postoperative course without the need for pharmacological, surgical, endoscopic or radiological interventions, allowed therapeutic regimes are drugs as antiemetics, antipyretics, analgesics, diuretics, electrolytes and physiotherapy; Grade 2 complications require pharmacological treatment with drugs other than those allowed for Grade 1 complications, blood transfusions and/or total parenteral nutrition; Grade 3 complications require surgical, endoscopic or radiological interventions; Grade 4 complications indicate life-threatening events including central nervous system complications requiring intermediate/intensive care unit management; and Grade 5 complications describe the death of a patient. Clavien-Dindo Grade 1-2 complications are
Table 2 Histopathology severity

\begin{tabular}{llll}
\hline & $\begin{array}{l}\text { Early, } \mathbf{n = I 3 8} \\
\mathbf{n}(\%)\end{array}$ & $\begin{array}{l}\text { Intermediate, } \mathbf{n = 2 9 7} \\
\mathbf{n}(\%)\end{array}$ & $\begin{array}{l}\text { Delayed, } \mathbf{n = 4 2 7} \\
\mathbf{n}(\%)\end{array}$ \\
\hline 0 & $41(29.7)$ & $155(52.2)$ & $311(72.8)$ \\
1 & $52(37.7)$ & $81(27.3)$ & $88(20.7)$ \\
2 & $29(21.0)$ & $47(15.8)$ & $22(5.2)$ \\
3 & $16(11.6)$ & $14(4.7)$ & $6(1.4)$ \\
\hline
\end{tabular}

Notes: Early: Surgery performed within 72 hours of the onset of symptoms. Intermediate: Surgery performed within 10 days on average from the onset of symptoms (for detailed description, see the "Results" section: descriptive data). Delayed: Surgery performed in symptom-free interval (6-12 weeks after the onset of symptoms). 0: Chronic inflammation. I: Acute erosive inflammation. 2: Acute inflammation with abscess, empyema, ulcero-phlegmonos changes or necrosis. 3: Acute inflammatory covered perforation, with/without peritonitis.

defined as mild, with Clavien-Dindo Grade 3-5 indicating severe complications.

Detailed data on the severity of complications in the Clavien-Dindo complication score are shown in Table 3. These findings correlate significantly $(P<0.0001)$ with the severity of histologic changes.

Clavien-Dindo complication scores differed significantly among the four groups (see Figure 1; Table 3). To adjust for main confounders and avoid a confounding bias, we used a stratified nonparametric analysis. Even after stratification by histology classification, severity of histologic changes and ASA-score, the Clavien-Dindo complication score was significantly different among the four groups $(P=0.022)$. The adjusted linear rank statistics showed a decrease in the complication score with values of 2.29 in the early group, 
Table 3 Clavien-Dindo complication score

\begin{tabular}{lllll}
\hline Clavien & $\begin{array}{l}\text { Early, } \mathbf{n = 1 3 8} \\
\mathbf{n}(\%)\end{array}$ & $\begin{array}{l}\text { Intermediate, } \mathbf{n = 2 9 7} \\
\mathbf{n}(\%)\end{array}$ & $\begin{array}{l}\text { Delayed, } \mathbf{n = 4 2 7} \\
\mathbf{n}(\%)\end{array}$ & $\begin{array}{l}\text { Elective, } \mathbf{n = 8 6} \text { I } \\
\mathbf{n}(\%)\end{array}$ \\
\hline 0 & $99(71.7)$ & $248(83.5)$ & $382(89.7)$ & $804(93.4)$ \\
1 & $7(5.1)$ & $17(5.7)$ & $13(3.1)$ & $28(3.3)$ \\
2 & $16(I 1.6)$ & $18(6.1)$ & $23(5.2)$ & $22(2.6)$ \\
3 & $1(0.7)$ & $6(2.0)$ & $4(1.0)$ & $4(0.5)$ \\
4 & II (8.0) & $7(2.4)$ & $5(1.2)$ & $3(0.3)$ \\
5 & $4(2.9)$ & $1(0.3)$ & $0(0.0)$ & $0(0.0)$ \\
\hline
\end{tabular}

Notes: Early: Surgery performed within 72 hours of the onset of symptoms. Intermediate: Surgery performed within 10 days on average from onset of symptoms (for detailed description, see the "Results" section: descriptive data). Delayed: Surgery performed in symptom-free interval (6-12 weeks after the onset of symptoms). Elective: Surgery performed electively in symptomatic cholecystolithiasis without signs of acute cholecystitis.

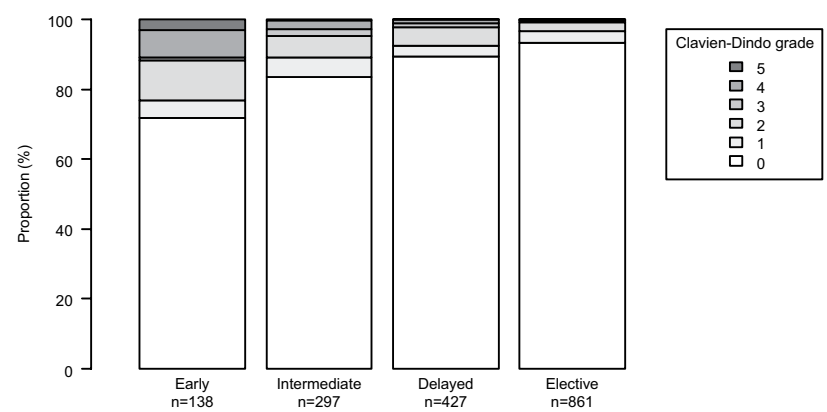

Figure I Clavien-Dindo complication score by the operation group.

0.48 in the intermediate group, -0.26 in the delayed group and -2.12 in the elective group. The results translate into a continuous decrease of the complication score from early over intermediate and delayed to the elective group.

\section{Discussion}

In contrast to previous published results, our retrospective study confirms the clinical impression that early cholecystectomy for acute cholecystitis has the highest risk rank when compared to an intermediate and a delayed approach.

We believe that the main reason why our results differ from previously published studies may be the lack of inclusion of severe cases of cholecystitis in those studies. ${ }^{10,11}$ In contrast to these studies, the early group in our study comprised a significant proportion of severe cases as documented by the pathology reports, which are considered as the diagnostic gold standard. ${ }^{13}$ The best way to define the severity of acute cholecystitis remains a matter of debate. The updated Tokyo guidelines (TG13) ${ }^{18}$ suggest the following grading with a linked therapeutic recommendation:

- Grade I (mild) acute cholecystitis: ELC is the preferred procedure.

- Grade II (moderate) acute cholecystitis: Early cholecystectomy is recommended in experienced centers. However, if patients have severe local inflammation, early gallbladder drainage (percutaneous or surgical) is indicated. Because early cholecystectomy may be difficult, medical treatment and delayed cholecystectomy are necessary.

- Grade III (severe) acute cholecystitis: Urgent management of organ dysfunction and the management of severe local inflammation by gallbladder drainage should be carried out. Delayed elective cholecystectomy should be performed when cholecystectomy is indicated.

This grading system was questioned by the 2016 World Society of Emergency Surgery guidelines, ${ }^{19}$ stating that the TG13 classification lacks clinical validation. Of note, there has been no randomized study addressing the surgical treatment according to the severity of acute cholecystitis to date. This could be due to the inability to randomize patients requiring emergency treatment. Our patients in the early group, which requires urgent intervention, by definition, could not have been randomized to an early or a delayed group in a randomized study.

With regard to mortality, we did not observe an increase of overall mortality in our study population compared to published results. Our observed mortality rate of $2.9 \%$ in our early group is comparable with the mortality rate of $2.8 \%$ in empyematous cholecystitis in the German health care system. ${ }^{20}$ In a different health care system (Medicare), a similar mortality rate of $3 \%$ has been reported in a high-risk population. ${ }^{21}$

The main limitation of our study is its retrospective nature. Group assignment of patients and choice of treatment plan were left to individual senior surgeon's choice with inherent bias. ${ }^{22,23}$ But as already discussed, we think that severe cases of acute cholecystitis (necrotizing, perforated, abscesses, gangrenous, empyematous) cannot be randomized to an early vs delayed approach trial because of the emergent nature of the disease. Thus, the inherent bias in the choice of treatment cannot be solved, since clear distinctions among mild, moderate and severe cases are not validated. 
Since the diagnostic impression of an experienced clinician appears more accurate than any single component of history, physical exam or lab data for the diagnosis of acute cholecystitis, ${ }^{24}$ the indication for the early, that is, emergency approach in our study is obviously based intuitively on clinical grounds and is supported by the later histologic findings in the cholecystectomy specimens.

An additional limitation of our study may lie in the limited control over the nature and quality of the baseline measurements. The existing data may be incomplete or inaccurate, or measured in ways that are not ideal for answering the research question. ${ }^{25}$ Otherwise, the audits of our record documentation prove a very high adherence to preset standards by physicians. That is why, we think that our data and the standardized parameters analyzed in our study are of a robust nature (eg, surgical procedure, histopathology, conversion rate, operation time, time of hospital stay), that is, these data are available in every case.

Another shortcoming of our study is the fact that clinical decision was not standardized, but left to the individual and bias opinion of each attending surgeon. This opens our study to individual surgeon's bias. This is particularly relevant as this was a single-institution study. On the other side, we can document a large effect size when comparing the mortality rate of the early and delayed groups. In this case, the GRADE system allows to upgrade the results of observational studies to the level of randomized controlled trials. ${ }^{26}$ Taken together, despite the mentioned limitations of our study, we think that our data are reflecting a real-world clinical setting.

\section{Conclusion}

The results demonstrate that delayed cholecystectomy can be performed safely in our hospital and may present advantages in outcomes compared to early and urgent intervention for acute cholecystitis. Furthermore, including severe cases, patients with an early and/or intermediate approach still have a relatively high risk of morbidity and mortality.

\section{Author contributions}

J Blythe and DrT Edwards-Lehr contributed to the data analyses, patient chart analysis and presentation of the raw data and revised the manuscript. J Blythe did the literature research and wrote the manuscript. Prof E Herrmann contributed to interpretation of the data and the statistical analysis, wrote the statistical part of the manuscript and revised the manuscript. Prof D Faust provided the data of the conservatively treated patients, contributed to interpretation of the data, and wrote and revised the manuscript. Prof S Falk provided the histopathologic exams and data and their interpretation, and wrote and revised the manuscript. Dr F Stockhausen did the literature research, contributed the web material, and wrote and revised the manuscript. Prof E Hanisch and Dr A Buia did the literature research, contributed to data interpretation, and wrote and revised the manuscript. All authors gave final approval of the version to be published; and agree to be accountable for all aspects of the work.

\section{Disclosure}

The authors report no conflicts of interest in this work.

\section{References}

1. Cholecystitis [webpage on the Internet]. London: BMJ Best Practice; 2018. Available from: https://bestpractice.bmj.com/topics/en-gb/78. Accessed September 19, 2018.

2. David GG, Al-Sarira AA, Willmott S, Deakin M, Corless DJ, Slavin JP. Management of acute gallbladder disease in England. Br J Surg. 2008;95(4):472-476.

3. Götzky K, Landwehr P, Jähne J. Epidemiology and clinical presentation of acute cholecystitis. Chirurg. 2013;84(3):179-184.

4. Kehr H. Die Praxis der Gallenwege-Chirurgie in Wort und Bild. I. Band: Die Vorbereitungen zu einer Operation an den Gallenwegen und die allgemeine Technik der Gallenwege-Chirurgie [The practice of surgery of the biliary tract - surgery in words and pictures (No.1) The preparation of a biliary tract operation and techniques in biliary tract surgery]. München: J. F. Lehmann's Verlag; 1913. German.

5. Graham HF, Hoefle ME, Milton E. Acute cholecystitis: the results of operation within forty-eight hours of the onset of symptoms. Ann Surg. 1938;108(5):874-876.

6. Gurusamy KS, Davidson C, Gluud C, Davidson BR. Early versus delayed laparoscopic cholecystectomy for people with acute cholecystitis. Cochrane Database Syst Rev. 2013;6:CD005440.

7. Cao AM, Eslick GD, Cox MR. Early cholecystectomy is superior to delayed cholecystectomy for acute cholecystitis: a meta-analysis. J Gastrointest Surg. 2015;19(5):848-857.

8. Cao AM, Eslick GD, Cox MR. Early laparoscopic cholecystectomy is superior to delayed acute cholecystitis: a meta-analysis of case-control studies. Surg Endosc. 2016;30(3):1172-1182.

9. Wu XD, Tian X, Liu MM, Wu L, Zhao S, Zhao L. Meta-analysis comparing early versus delayed laparoscopic cholecystectomy for acute cholecystitis. Br J Surg. 2015;102(11):1302-1313.

10. Gutt CN, Encke J, Köninger J, et al. Acute cholecystitis: early versus delayed cholecystectomy, a multicenter randomized trial (ACDC study, NCT00447304). Ann Surg. 2013;258(3):385-393.

11. de Mestral C, Rotstein OD, Laupacis A, et al. Comparative operative outcomes of early and delayed cholecystectomy for acute cholecystitis: a population-based propensity score analysis. Ann Surg. 2014;259(1): $10-15$.

12. Pitt HA. Patient value is superior with early surgery for acute cholecystitis. Ann Surg. 2014;259(1):16-17.

13. Strasberg SM. Clinical practice. Acute calculous cholecystitis. $N$ Engl J Med. 2008;358(26):2804-2811.

14. von Elm E, Altman DG, Egger M, et al. The Strengthening the Reporting of Observational Studies in Epidemiology (STROBE) statement: guidelines for reporting observational studies. Int J Surg. 2014;12(12):1495-1499.

15. Dindo D, Demartines N, Clavien PA. Classification of surgical complications: a new proposal with evaluation in a cohort of 6336 patients and results of a survey. Ann Surg. 2004;240(2):205-213.

16. Wawra N, Buia A, Hanisch E. Development of single-port cholecystectomy: results of a case-control study matched to one surgeon. Open Access Surg. 2012;5:33-38. 
17. Hothorn T, Hornik K, van de Wiel MA, Zeileis A. A Lego system for conditional inference. Am Stat. 2006;60(3):257-263.

18. Yamashita Y, Takada T, Strasberg SM, et al. TG13 surgical management of acute cholecystitis. J Hepatobiliary Pancreat Sci. 2013;20(1): 89-96.

19. Ansaloni L, Pisano M, Coccolini F. WSES guidelines on acute calculous cholecystitis. World J Emerg Surg. 2016;2016(11):1-23.

20. Ambe PC, Jansen S, Macher-Heidrich S, Zirngibl H. Surgical management of empyematous cholecystitis: a register study of over 12,000 cases from a regional quality control database in Germany. Surg Endosc. 2016;30(12):5319-5324.

21. Fry DE, Pine M, Nedza S, Locke D, Reband A, Pine G. Hospital outcomes in inpatient laparoscopic cholecystectomy in Medicare patients. Ann Surg. 2017;265(1):178-184.
22. Freemantle N, Marston L, Walters K, Wood J, Reynolds MR, Petersen I. Making inferences on treatment effects from real world data: propensity scores, confounding by indication, and other perils for the unwary in observational research. BMJ. 2013;347:f6409-f6409.

23. Unverzagt $S$, Prondzinsky R, Peinemann F. Single-center trials tend to provide larger treatment effects than multicenter trials: a systematic review. J Clin Epidemiol. 2013;66(11):1271-1280.

24. Acute cholecystitis. Available from: http://www.dynamed.com/topics/ dmp AN T114758/Acute-cholecystitis. Accessed March 25, 2018

25. Hulley SB, Cummings SR, Browner WS, Grady DG, Newman TB Designing Clinical Research. 4th ed. Philadelphia, PA: Lippincott Williams \& Wilkins, a Wolters Kluwer business; 2013.

26. Howick J. The Philosophy of Evidence-based Medicine. San Francisco, CA: Wiley-Blackwell; 2011.
Pragmatic and Observational Research

\section{Publish your work in this journal}

Pragmatic and Observational Research is an international, peer-reviewed, open access journal that publishes data from studies designed to reflect more closely medical interventions in real-world clinical practice compared with classical randomized controlled trials (RCTs). The manuscript management system is completely online and includes a very quick and fair peer-review

\section{Dovepress}

system. Visit http://www.dovepress.com/testimonials.php to read real quotes from published authors.

Submit your manuscript here: https://www.dovepress.com/pragmatic-and-observational-research-journal 